Research Article

\title{
Dyons, Superstrings, and Wormholes: Exact Solutions of the Non-Abelian Dirac-Born-Infeld Action
}

\author{
Edward A. Olszewski \\ Department of Physics, University of North Carolina at Wilmington, Wilmington, NC 28403-5606, USA \\ Correspondence should be addressed to Edward A. Olszewski; olszewski@uncw.edu
}

Received 14 April 2015; Accepted 16 July 2015

Academic Editor: Anastasios Petkou

Copyright (C) 2015 Edward A. Olszewski. This is an open access article distributed under the Creative Commons Attribution License, which permits unrestricted use, distribution, and reproduction in any medium, provided the original work is properly cited. The publication of this article was funded by SCOAP ${ }^{3}$.

\begin{abstract}
We construct dyon solutions on coincident $D 4$-branes, obtained by applying $T$-duality transformations to type I SO(32) superstring theory in 10 dimensions. These solutions, which are exact, are obtained from an action comprising the non-Abelian Dirac-BornInfeld action and a Wess-Zumino-like action. When one spatial dimension of the D4-branes is taken to be vanishingly small, the dyons are analogous to the 't Hooft/Polyakov monopole residing in a $3+1$-dimensional spacetime, where the component of the Yang-Mills potential transforming as a Lorentz scalar is reinterpreted as a Higgs boson transforming in the adjoint representation of the gauge group. Applying a $T$-duality transformation to the vanishingly small spatial dimension, we obtain a collection of $D 3$ branes, not all of which are coincident. Two of the D3-branes, distinct from the others, acquire intrinsic, finite curvature and are connected by a wormhole. The dyons possess electric and magnetic charges whose values on each D3-brane are the negative of one another. The gravitational effects, which arise after the $T$-duality transformation, occur despite the fact that the action of the system does not explicitly include the gravitational interaction. These solutions provide a simple example of the subtle relationship between the Yang-Mills and gravitational interactions, that is, gauge/gravity duality.
\end{abstract}

\section{Introduction}

Theoretically appealing but experimentally elusive, the magnetic monopole has captured the interest of the physics community for more than eight decades. The magnetic monopole (an isolated north or south magnetic pole) is conspicuously absent from the Maxwell theory of electromagnetism. In 1931, Dirac showed that the magnetic monopole can be consistently incorporated into the Maxwell theory with virtually no modification to the theory [1]. In addition, Dirac demonstrated that the existence of a single magnetic monopole necessitates not only that electric charge be quantized but also that the electric and magnetic couplings be inversely proportional to each other, the first suggestion of the so-called weak/strong duality. Subsequently, 't Hooft [2] and Alexander Polyakov showed that, within the context of the spontaneously broken Yang-Mills gauge theory $S O(3)$, topological magnetic monopole solutions of finite mass must necessarily exist. Furthermore, these solutions possess an internal structure and also exhibit the same weak/strong duality discovered by Dirac. Consequently, Montonen and Olive conjectured that there exists an exact weak/strong electromagnetic duality for the spontaneously broken $\mathrm{SO}(3)$ gauge theory [3]. More recently, this conjecture has become credible within the broader context of $N=2$ or $N=4$ SuperYang-Mills theories. Despite the lack of experimental evidence for the existence of magnetic monopoles, physicists still remain optimistic of their existence. Indeed, Guth proposed the inflationary model of the universe, in part, to explain why magnetic monopoles have escaped discovery [4].

The focus of our investigation is electrically charged magnetic monopole (dyon) solutions within the context of superstring theory. In Section 2, we construct dyon solutions which are exact and closed to first order in the string theory length scale. We, first, begin with a type I $S O(32)$ string theory in ten dimensions, six of the spatial dimensions being compact but arbitrarily large. We, then, apply the group of $T$-duality transformations to five of the compact spatial dimensions to obtain $16 \mathrm{D} 4$-branes, some of which are coincident. The five $T$-dualized dimensions of each $D 4$-brane 
constitute the internal dimensions of a $4+1$-dimensional spacetime. Making an appropriate ansatz, we obtain dyon solutions residing on the D4-branes. The solutions are based on an action which includes coupling of the D4-branes to NS-NS closed strings, the non-Abelian Dirac-Born-Infeld action, and coupling to $R-R$ closed strings, a Wess-Zuminolike action. We next apply a $T$-duality transformation to the D4-branes, resulting in a collection of D3-branes, some of which are coincident and two of which are connected by a wormhole. Finally, we interpret the dyon solutions in the context of gauge/gravity duality.

Because of the differences in the literature among the systems of units, sign conventions, and so forth, we present in Appendix A the conventions chosen by us so that direct comparisons can be made between our results and those of other authors.

\section{Dyons and Dimensional Reduction of Type I SO(32) Theory}

In this section, we construct dyon solutions based on superstring theory. We begin with type I SO(32) superstring theory in ten dimensions [5], six of the spatial dimensions of which are compact. Next, we apply the group of $T$-duality transformations to five of the compact dimensions letting the size, $R$, of the dimensions become vanishingly small; that is, $R \rightarrow 0$. These five dimensions are the internal dimensions of spacetime. Strictly speaking, spacetime consists of 16 D4-branes, bounded by $2^{5}$ orientifold hyperplanes. Each of the D4-branes comprises four spatial dimensions, three unbounded and one compact. In what follows, we assume that none of the $D$-branes are close to the orientifold hypersurfaces. Thus, the theory describing the closed strings in the vicinity of any of the D4-branes is type II oriented, rather than type II unoriented. In this particular case, since we have applied the $T$-duality transformation to an odd number of dimensions, the closed string theory is the type IIa oriented theory. Furthermore, each end of an open string must be attached to a D4-brane, which may be the same D4-brane or two different $D 4$-branes. If we assume that the number of coincident D4-branes is $n(2 \leq n \leq 16)$, then a $U(n)$ gauge group is associated with the open strings attached to the coincident D4-branes. Given these prerequisite conditions, we now construct dyon solutions which reside on these coincident D4-branes. These solutions are derived from the $D$-brane action comprising two parts, the Dirac-Born-Infeld action, $S_{\mathrm{DBI}}$, which couples NS-NS closed strings to the D4brane and the Wess-Zumino-like action, $S_{\mathrm{WZ}}$, which couples $R-R$ closed strings to the D4-brane.

2.1. Dyon Solutions on D4-Branes. The dyon solutions are obtained from the equations of motion derived from the action, $S$, which describes the coupling of closed string fields to a general $D p$-brane (which in our case is $p=4$ ). The action is [6] where

$$
\begin{aligned}
& S_{\mathrm{DBI}}=-\tau_{p} \int_{\mathscr{M}_{p+1}} \operatorname{STr}\left\{e^{-\Phi}[(-1)\right. \\
& \left.\left.\cdot \operatorname{det}\left(G_{A B}+B_{A B}+2 \pi \alpha^{\prime} F_{A B}\right)\right]^{1 / 2}\right\}, \\
& S_{\mathrm{WZ}}=\mu_{p} \int_{\mathscr{M}_{p+1}}\left[\sum_{p^{\prime}} C_{\left(p^{\prime}+1\right)}\right] \wedge \operatorname{Tr} e^{2 \pi \alpha^{\prime} F+B} .
\end{aligned}
$$

Here, $\tau_{p}$ is the physical tension of the $D p$-brane, and $\mu_{p}$ is its R-R charge (see Appendix B for a discussion of the relationships among the various string parameters).

The dyon solutions are based on the following ansatz. The dilaton background, $\Phi$, is constant:

$$
\Phi=\Phi_{0}
$$

And background field $B$ vanishes:

$$
B_{A B}=0 \quad(A, B=0 \cdots 4) .
$$

The metric $G$ is given by

$$
G_{A B}=\widetilde{G}_{A B} I_{n},
$$

where, for our purposes, $\widetilde{G}_{A B}$ is restricted so that $\widetilde{G}_{00}=-1$ and $\widetilde{G}_{44=1}$.

For $p=4$, we can reexpress the determinant in (2) as

$$
\begin{aligned}
\operatorname{det}\left(G_{A B}+2 \pi \alpha^{\prime} F_{A B}\right)=\operatorname{det}\left(\widetilde{G}_{A B}\right)\left[I_{n}+\frac{2 \pi \alpha^{\prime}}{2 !} F_{A B} F^{A B}\right. \\
\left.-\frac{\left(2 \pi \alpha^{\prime}\right)^{2}}{(5-4) !}\left(3^{2} \cdot 1^{2}\right)^{*}(F \wedge F)_{E}^{*}(F \wedge F)^{E}\right]
\end{aligned}
$$

where

$$
{ }^{*}(F \wedge F)_{E}=\frac{\sqrt{\left|\operatorname{det}\left(\widetilde{G}_{A B}\right)\right|}}{4 !} F^{A B} F^{C D} \epsilon_{A B C D E} .
$$

See Appendix C, (C.21), for further details.

The term $I_{n}$ is the $n$-dimensional identity matrix. The value of $n$ is the dimension of the group $U(n)$ associated with the gauge fields residing on the $D 4$-branes. All $R-R$ potentials vanish, except for the one-form potential $C_{(1)}$, which is a constant background field,

$$
C_{(1)}=C_{4} d x^{4},
$$

for some constant value $C_{4}$. The gauge field, $F$, is obtained from the gauge potential $A\left(A=A_{E} d x^{E}\right)$, where

$$
\begin{aligned}
& A_{\mu}=A_{\mu}\left(x^{i}\right), \quad(\mu=0 \cdots 3 ; i=1 \cdots 3), \\
& A_{4}=A_{4}\left(x^{i}\right) .
\end{aligned}
$$

$$
S=S_{\mathrm{DBI}}+S_{\mathrm{WZ}}
$$


Note that the gauge potentials are static; that is, they do not depend on time, $x^{0}$, and also do not depend on the spatial coordinate $x^{4}$. The gauge field $F\left(F=F_{A B} d x^{A} \wedge d x^{B}\right)$, a Lie algebra-valued two-form, is given by

$$
F=d A-i A \wedge A \text {. }
$$

(See Appendix A.) The components of the potentials $A_{0}$ and $A_{4}$ are constrained in accordance with the condition

$$
A_{0} \wedge A_{4}=0
$$

so that $F_{04}=0$. To facilitate its interpretation, we express $F_{A B}$ as a five-dimensional matrix which is explicitly partitioned into electric and magnetic fields which reside in four-dimensional spacetime and an additional component of the magnetic field which resides in the additional space dimension; that is,

$$
F_{A B}=\left(\begin{array}{ccccc}
0 & E_{1} & E_{2} & E_{3} & 0 \\
-E_{1} & 0 & B_{3} & -B_{2} & -\mathscr{D}_{1} A_{4} \\
-E_{2} & -B_{3} & 0 & B_{1} & -\mathscr{D}_{2} A_{4} \\
-E_{3} & B_{2} & -B_{1} & 0 & -\mathscr{D}_{3} A_{4} \\
0 & \mathscr{D}_{1} A_{4} & \mathscr{D}_{2} A_{4} & \mathscr{D}_{3} A_{4} & 0
\end{array}\right) .
$$

We are seeking dyon solutions. Therefore, with foresight, we make the following assumptions:

$$
\begin{aligned}
E_{i} & \equiv F_{0 i}^{a} T_{a}=F_{0 i}^{(i)} T_{(i)}, \\
B_{i} & \equiv \frac{1}{2} \epsilon_{i}^{j k} F_{j k}^{a} T_{a}=\frac{1}{2} \epsilon_{i}^{j k} F_{j k}^{(i)} T_{(i)}, \\
\mathscr{D}_{i} A_{4} & \equiv\left(\partial_{i} A_{4}-i A_{i} \wedge A_{4}\right)^{a} T_{a}=F_{4 i}^{a} T_{a}=F_{4 i}^{(i)} T_{(i)} .
\end{aligned}
$$

The parenthetical index $(i)$ indicates that there is no summation of that index; however, if an expression contains two indices $i$ without parentheses, then summation of these two indices is implied. Furthermore, each matrix element in (13) includes a generator of $U(n)$; for example, $E_{i}=E_{i}^{(i)} T_{(i)}$. Because we are seeking dyon solutions, we may assume without loss of generality that each $T_{(i)}$ is a generator in the fundamental representation of a local $U(1) \times S U(2)$ subgroup of $S U(n)$ (see (44a), (44b), (44c), and (44d)).

The action, (2), can be more straightforwardly interpreted from the perspective of four-dimensional spacetime. Since the action does not depend on the coordinate $x^{4}$, we can trivially eliminate $x^{4}$ from the action by integrating the $x^{4}$ coordinate. As a result of the integration, the tension of the $D 4$-brane, $\tau_{4}$, and the Yang-Mills coupling constant, $g_{D 4}$, are replaced by those of the D3-brane, $\tau_{3}$ and $g_{D 3}$ (see (B.3) and (B.5)). Let the size, $R_{4}$, of the $x^{4}$-dimension become vanishingly small; that is, $R_{4} \rightarrow 0$. Then, the field $A_{4}$ becomes a Lorentz scalar transforming as the adjoint representation of the gauge group, and (14) gives the covariant derivative of $A_{4}$. From the perspective of four spacetime dimensions, $A_{4}$ assumes the role of a Higgs boson transforming as the adjoint representation of the gauge group.
Substituting (4)-(6) and (13) into (1) and then integrating the $x^{4}$ coordinate, we obtain

$$
S_{\mathrm{DBI}}=\int d^{3+1} \xi \mathscr{L}_{\mathrm{DBI}},
$$

where

$$
\begin{aligned}
& \mathscr{L}_{\text {DBI }} \\
& =-\frac{1}{\left(2 \pi \alpha^{\prime}\right)^{2} g_{D 3}^{2}} \operatorname{STr}\left[\left\{\left|\operatorname{det}\left(G_{A B}+2 \pi \alpha^{\prime} F_{A B}\right)\right|^{1 / 2}\right\}\right] \\
& =-\frac{\sqrt{\left|\operatorname{det}\left(\widetilde{G}_{i j}\right)\right|}}{\left(2 \pi \alpha^{\prime}\right)^{2} g_{D 3}^{2}} \operatorname{STr}\left\{\mathscr{L}^{\prime}\right\} .
\end{aligned}
$$

The function $\mathscr{L}^{\prime}$ is defined as

$$
\begin{aligned}
\mathscr{L}^{\prime} & =\left\{I_{n}-\left(2 \pi \alpha^{\prime}\right)^{2}\left(E \cdot E-B \cdot B-\mathscr{D} A_{4} \cdot \mathscr{D} A_{4}\right)\right. \\
& +\left(2 \pi \alpha^{\prime}\right)^{4}\left(B \cdot \mathscr{D} A_{4}\right)^{2}-\left(2 \pi \alpha^{\prime}\right)^{4}(E \cdot B)^{2} \\
& \left.-\left(2 \pi \alpha^{\prime}\right)^{4}\left(E \times \mathscr{D} A_{4}\right) \cdot\left(E \times \mathscr{D} A_{4}\right)\right\}^{1 / 2} .
\end{aligned}
$$

We have used the fact that $\sqrt{\left|\operatorname{det}\left(\widetilde{G}_{i j}\right)\right|}=\sqrt{\left|\operatorname{det}\left(\widetilde{G}_{A B}\right)\right|}$. In (16), the ordering of the generators of the algebra, $T_{a}$, corresponds with the order of the fields as they appear in the equation; for example,

$$
\begin{gathered}
\left\{B \cdot \mathscr{D} A_{4}\right\}^{2}=\left\{B \cdot \mathscr{D} A_{4}\right\}\left\{B \cdot \mathscr{D} A_{4}\right\} \\
=\left\{\widetilde{G}^{i j} B_{i}^{(i)} T_{(i)}\left(\mathscr{D}_{j} A_{4}\right)^{(j)} T_{(j)}\right\} \\
\cdot\left\{\widetilde{G}^{i j} B_{i}^{(i)} T_{(i)}\left(\mathscr{D}_{j} A_{4}\right)^{(j)} T_{(j)}\right\} .
\end{gathered}
$$

Note that "STr" indicates that the trace is calculated symmetrically; that is, the trace is symmetrized with respect to all gauge indices $[6,7]$. The implication is that the evaluation of the trace requires that after the expansion of (16) in powers of the field strengths, all orderings of the field strengths are included with equal weight; that is, products of $T_{a}$ are replaced by their symmetrized sum, before the trace is evaluated. This is discussed in detail in $[6,7]$.

In (16), the dot product and cross product of two 3vectors, for example, $E$ and $\mathscr{D} A_{4}$, are defined as $E \cdot \mathscr{D} A_{4}=$ $\widetilde{G}^{i j} E_{i} \mathscr{D}_{j} A_{4}$ and $\left(E \times \mathscr{D} A_{4}\right)_{i}=\varepsilon_{i}^{j k} E_{j} \mathscr{D}_{k} A_{4}$.

In obtaining (16), we have reexpressed the dilaton $\Phi$, on a D4-brane, in terms of the dilaton $\Phi^{\prime}$, on a D3-brane, both of which are related by a $T$-duality transformation in the $x^{4}$-dimension. Specifically, $\Phi$ and $\Phi^{\prime}$ are related by $e^{\Phi^{\prime}}=$ $\alpha^{1 / 2} e^{\Phi} / R_{4}$. The constant dilaton background $\phi_{0}$ has been incorporated into the physical tension $\tau_{p}$ (see Appendix B).

Substituting (5), (9), (6), and (13) into (3), we obtain

$$
S_{\mathrm{WZ}}=\frac{\mu_{4}}{2 !} \int_{\mathscr{M}_{5}} C_{(1)} \wedge \operatorname{Tr}\left\{2 \pi \alpha^{\prime} F \wedge 2 \pi \alpha^{\prime} F\right\} .
$$


Integrating the $x^{4}$ coordinate in (19), we obtain

$$
S_{\mathrm{WZ}}=\int d^{3+1} \xi \mathscr{L}_{\mathrm{WZ}}
$$

where

$$
\begin{aligned}
\mathscr{L}_{\mathrm{WZ}} & =\frac{\theta}{4 \pi^{2}} \operatorname{Tr}\{F \wedge F\}=\sqrt{\left|\operatorname{det}\left(\widetilde{G}_{i j}\right)\right|} \frac{\theta}{4 \pi^{2}} \operatorname{Tr}\{E \cdot B\} \\
& =\sqrt{\left|\operatorname{det}\left(\widetilde{G}_{i j}\right)\right|} \frac{\theta}{4 \pi^{2}} E^{i(i)} B_{i}^{(i)} \operatorname{STr}\left\{T_{(i)} T_{(i)}\right\} \\
& =\sqrt{\left|\operatorname{det}\left(\widetilde{G}_{i j}\right)\right|} \frac{\theta}{8 \pi^{2}} E^{i(i)} B_{i}^{(i)}
\end{aligned}
$$

where $E^{i(i)}=F^{0 i(i)} T_{(i)}$. Here,

$$
\theta \equiv \frac{C_{4}}{2 !} \frac{2 \pi R_{4}}{\alpha^{\prime 1 / 2}}
$$

In obtaining (21), we have explicitly evaluated $\mu_{4}$ using (B.4). Equation (21) is associated with the Witten effect. Witten has demonstrated that adding term (21) to the Lagrangian of Yang-Mills theory does not alter the classical equations of motion but does alter the electric charge quantization condition in the magnetic monopole sector of the theory $[5,8,9]$. In summary, the action, $S$, for the $D 4$-brane is given by

$$
S=\int d^{3+1} \xi \mathscr{L}
$$

where

$$
\mathscr{L}=\mathscr{L}_{\mathrm{DBI}}+\mathscr{L}_{\mathrm{WZ}}
$$

The equations of motion which are obtained from (23) are

$$
\mathscr{D}_{\mu} P^{\mu \nu}=0 \text {, }
$$

where

$$
P_{\mu \nu}^{a}=\frac{\partial \mathscr{L}}{\partial F^{\mu \nu a}} .
$$

In addition, the fields $F_{\mu \nu}^{a}$ satisfy the Bianchi identity

$$
\mathscr{D}_{[\alpha} F_{\beta \gamma]}^{a}=0 \text {. }
$$

To facilitate the ensuing analysis, we transform the Lagrangian density, $\mathscr{L}$, to the Hamiltonian density, $\mathscr{H}$, using the Legendre transformation

where

$$
\mathscr{H}=\operatorname{STr}\left\{P_{0} \cdot E-\mathscr{L}\right\},
$$

$$
\begin{aligned}
P_{0 i}^{(i)} \equiv & \frac{\partial \mathscr{L}}{\partial E^{i(i)}} \\
= & -\frac{\sqrt{\left|\operatorname{det}\left(\widetilde{G}_{i j}\right)\right|}}{\left(2 \pi \alpha^{\prime}\right)^{2} g_{D 3}^{2}} \operatorname{Sr}\left\{\frac{X_{i}^{(i)}}{\mathscr{L}^{\prime}}\right\} \\
& +\sqrt{\left|\operatorname{det}\left(\widetilde{G}_{i j}\right)\right|} \frac{\theta}{4 \pi^{2}} B_{i}^{(i)} \operatorname{STr}\left\{T_{(i)} T_{(i)}\right\},
\end{aligned}
$$

where

$$
\begin{aligned}
X_{i}^{(i)}= & E_{i} T_{(i)}+(E \cdot B) T_{(i)} B_{i} \\
& +\left(\mathscr{D} A_{4} \times\left[E \times \mathscr{D} A_{4}\right]\right)_{i} T_{(i)}
\end{aligned}
$$

After performing detailed calculations, we obtain

$$
\mathscr{H}=\frac{\sqrt{\left|\operatorname{det}\left(\widetilde{G}_{i j}\right)\right|}}{\left(2 \pi \alpha^{\prime}\right)^{2} g_{D 3}^{2}} \operatorname{STr}\left\{\mathscr{H}^{\prime}\right\}
$$

where

$$
\begin{aligned}
\mathscr{H}^{\prime} & =\left\{I_{n}+\left(2 \pi \alpha^{\prime}\right)^{2}\left(P_{0} \cdot P_{0}+B \cdot B+\mathscr{D} A_{4} \cdot \mathscr{D} A_{4}\right)\right. \\
& +\left(2 \pi \alpha^{\prime}\right)^{4}\left(\left[B \cdot \mathscr{D} A_{4}\right]^{2}+\left[P_{0} \cdot \mathscr{D} A_{4}\right]^{2}\right. \\
+ & \left.\left.\frac{\left[P_{0} \times B\right]^{2}+\left[\mathscr{D} A_{4} \times\left(P_{0} \times B\right)\right]^{2}}{\left(2 \pi \alpha^{\prime}\right)^{-2} I_{n}+\mathscr{D} A_{4} \cdot \mathscr{D A} A_{4}}\right)\right\}^{1 / 2} .
\end{aligned}
$$

The electric field $E_{i}^{(i)}$ can be expressed as a function of $P_{0 i}^{(i)}$ :

$$
E_{i}^{(i)}=\frac{\partial \mathscr{H}}{\partial P_{0 i}^{(i)}}=\frac{\sqrt{\left|\operatorname{det}\left(\widetilde{G}_{i j}\right)\right|}}{\left(2 \pi \alpha^{\prime}\right)^{2} g_{D 3}^{2}} \operatorname{STr}\left\{\frac{Y_{i}^{(i)}}{\mathscr{H}^{\prime}}\right\} .
$$

The term $Y_{i}^{(i)}$ is given by

$$
\begin{aligned}
Y_{i}^{(i)} & =\left(2 \pi \alpha^{\prime}\right)^{2} P_{0 i} T_{(i)}+\left(2 \pi \alpha^{\prime}\right)^{4} \\
& \cdot\left[\mathscr{D}_{i} A_{4} T_{(i)}\left(P_{0} \cdot \mathscr{D} A_{4}\right)\right. \\
& +\left(B \times\left(P_{0} \times B\right)\right)_{i} T_{(i)} \\
& \left.-\frac{\left(B \times \mathscr{D} A_{4}\right)_{i} T_{(i)}\left(P_{0} \cdot\left(B \times \mathscr{D} A_{4}\right)\right)}{\left(2 \pi \alpha^{\prime}\right)^{-2} I_{n}+\mathscr{D} A_{4} \cdot \mathscr{D} A_{4}}\right] .
\end{aligned}
$$

We seek dyon solutions which are BPS states, that is, whose energy $\mathscr{E}\left(\mathscr{E}=\int d^{3} \xi \mathscr{H}\right)$ is a local minimum. First, we reexpress $\mathscr{H}$ 


$$
\begin{aligned}
\mathscr{H} & =\frac{\sqrt{\left|\operatorname{det}\left(\widetilde{G}_{i j}\right)\right|}}{\left(2 \pi \alpha^{\prime}\right)^{2} g_{D 3}^{2}} \operatorname{STr}\left[\left\{\left[I_{n}+\left(2 \pi \alpha^{\prime}\right)^{2}\left(\cos \phi P_{0} \cdot \mathscr{D} A_{4}+\sin \phi B \cdot \mathscr{D} A_{4}\right)\right]^{2}\right.\right. \\
& +\left(2 \pi \alpha^{\prime}\right)^{2}\left[\left(\sin \phi P_{0} \cdot \mathscr{D} A_{4}-\cos \phi B \cdot \mathscr{D} A_{4}\right)^{2}+\left(P_{0}-\cos \phi \mathscr{D} A_{4}\right)^{2}+\left(B-\sin \phi \mathscr{D} A_{4}\right)^{2}\right] \\
& \left.\left.+\left(2 \pi \alpha^{\prime}\right)^{4} \frac{\left[P_{0} \times B\right]^{2}+\left[\mathscr{D} A_{4} \times\left(P_{0} \times B\right)\right]^{2}}{\left(2 \pi \alpha^{\prime}\right)^{-2} I_{n}+\mathscr{D} A_{4} \cdot \mathscr{D} A_{4}}\right\}^{1 / 2}\right] .
\end{aligned}
$$

The mixing angle, $\psi$, between the electric and magnetic fields of the dyon is defined as

$$
\tan \psi=\frac{g_{m}}{g_{e}} .
$$

The quantities $g_{m}$ and $g_{e}$ are the electric and magnetic charges, respectively, of the dyon. The energy, $\mathscr{E}$, is minimized by constraining the dyon solutions to satisfy

$$
\begin{gathered}
P_{0}=\cos \psi \mathscr{D} A_{4}, \\
B=\sin \psi \mathscr{D} A_{4} .
\end{gathered}
$$

In (35), the second and third squared terms are zero as a consequence of the constraint. Since $P_{0} \propto B$, the fourth squared term is also zero by virtue of

$$
\begin{aligned}
& \left(P_{0} \times B\right)^{k} \\
& \quad=\frac{P_{0 i}^{(i)} B_{0 j}^{(j)}-P_{0 j}^{(j)} B_{0 i}^{(i)}}{2 !}\left(T_{(i)} T_{(j)} \varepsilon_{i j}^{k}-i f_{i j}^{(k)} T_{(k)}\right) .
\end{aligned}
$$

Thus, $\mathscr{H}$ simplifies so that the energy is

$$
\begin{aligned}
\mathscr{E} & =\frac{1}{\left(2 \pi \alpha^{\prime}\right)^{2} g_{D 3}^{2}} \int d^{3} \xi \sqrt{\left|\operatorname{det}\left(\widetilde{G}_{i j}\right)\right|} \operatorname{Tr}\left[I_{n}\right. \\
& \left.+\left(2 \pi \alpha^{\prime}\right)^{2}\left(\cos \phi P_{0} \cdot \mathscr{D} A_{4}+\sin \phi B \cdot \mathscr{D} A_{4}\right)\right] .
\end{aligned}
$$

Substituting (37a) and (37b) into (32) through (34) and using (39), we find

$$
E=P_{0}
$$

In (39), there are two terms which contribute to the mass of the system. The first term within the trace, that is, $I_{n}$, corresponds to the volume of each coincident D4-brane (or $D 3$-brane), which is infinite because the $D$-branes are not compact. The second term, by virtue of the equations of motion, (26), and the Bianchi identity, (27), can be expressed as a divergence and is therefore a topological invariant. The second term corresponds to the mass of the dyon and is proportional to $\sqrt{g_{e}^{2}+g_{m}^{2}}$ as discussed below.

The solutions to (25) and (27) can be straightforwardly obtained from the dyon solutions derived in [10]. Adapting the notation of [10] to the notation used here, we express the vector potential $A,(10)$, in the form (in accordance with our conventions, the Yang-Mills coupling constant appears explicitly in the Lagrangian (A.1). In $[8,10]$, the coupling constant has been incorporated into the Yang-Mills fields. Thus, to compare results here with those in the references, the fields $A$ and related fields should be divided by $g_{D 3}$ )

$$
\begin{aligned}
A= & A_{\mu} d x^{\mu}+A_{4} d x^{4} \\
= & \cos \psi S(r) g_{D 3} v \alpha_{1} T_{r} d t \\
& +W(r)\left[T_{\theta} \sin (\theta) n d \phi-T_{\phi} d \theta\right] \\
& +g_{D 3} v\left[\alpha_{2} T_{\perp}+Q(r) \alpha_{1} T_{r}\right] d x^{4},
\end{aligned}
$$

where $v$ is an arbitrary constant. For the Lie group $S U(n)$

$$
\begin{aligned}
& \alpha_{1}=\sqrt{\frac{n}{2(n-1)}} \\
& \alpha_{2}=-\sqrt{\frac{n-2}{2(n-1)}} .
\end{aligned}
$$

Here, $T_{i}(i=r, \theta, \phi)$ constitute a representation of the $S U(2)$ subalgebra and $T_{\perp}$ commutes with each $T_{i}$. The quantities $r, \theta, \phi$ are the spherical polar coordinates in three dimensions. The elements $T_{r}, T_{\theta}, T_{\phi}$ are related to $T_{x}, T_{y}, T_{z}$ :

$$
\begin{aligned}
& T_{r}=T_{x} \sin \theta \cos n_{m} \phi+T_{y} \sin \theta \sin n_{m} \phi+T_{z} \cos \theta, \\
& T_{\theta}=T_{x} \cos \theta \cos n_{m} \phi+T_{y} \cos \theta \sin n_{m} \phi-T_{z} \sin \theta, \\
& T_{\phi}=-T_{x} \sin n_{m} \phi+T_{y} \cos n_{m} \phi .
\end{aligned}
$$

For $S U(n)$, the $n$-dimensional matrices $T_{x}, T_{y}, T_{z}$, and $T_{\perp}$ are given by

$$
T_{x}=\frac{1}{2}\left(\begin{array}{ccccc}
0 & \cdots & 0 & 0 & 0 \\
\vdots & \ddots & \vdots & \vdots & \vdots \\
0 & \cdots & 0 & 0 & 0 \\
0 & \cdots & 0 & 0 & 1 \\
0 & \cdots & 0 & 1 & 0
\end{array}\right),
$$




$$
\begin{aligned}
& T_{y}=\frac{1}{2}\left(\begin{array}{ccccc}
0 & \cdots & 0 & 0 & 0 \\
\vdots & \ddots & \vdots & \vdots & \vdots \\
0 & \cdots & 0 & 0 & 0 \\
0 & \cdots & 0 & 0 & -i \\
0 & \cdots & 0 & i & 0
\end{array}\right) \text {, } \\
& T_{z}=\frac{1}{2}\left(\begin{array}{ccccc}
0 & \cdots & 0 & 0 & 0 \\
\vdots & \ddots & \vdots & \vdots & \vdots \\
0 & \cdots & 0 & 0 & 0 \\
0 & \cdots & 0 & 1 & 0 \\
0 & \cdots & 0 & 0 & -1
\end{array}\right) \text {, } \\
& T_{\perp}=\frac{1}{2 \sqrt{n(n-2)}}\left(\begin{array}{ccccc}
-2 & 0 & \cdots & 0 & 0 \\
0 & \ddots & \vdots & \vdots & \vdots \\
\vdots & \cdots & -2 & 0 & 0 \\
0 & \cdots & 0 & n-2 & 0 \\
0 & \cdots & 0 & 0 & n-2
\end{array}\right) .
\end{aligned}
$$

$T_{x}, T_{y}, T_{z}$, and $T_{\perp}$ are suitable linear combinations of specific elements of the Cartan subalgebra of $S U(n)$ (see [10] for details). The value of the integer $n_{m}$ in (43a), (43b), and $(43 \mathrm{c})$ is the integer multiple of the fundamental unit of dyon's magnetic charge.

These results differ from those of [10]. For a direct comparison, first replace the azimuthal angle, $\phi$, in [10] with $\phi^{\prime}$ and extend the domain from $[0,2 \pi]$ to $\left[0,2 \pi n_{m}\right]$; that is, $\phi^{\prime} \in\left[0,2 \pi n_{m}\right]$. Now, perform the change of variables $\phi^{\prime}=$ $n_{m} \phi$ to the dyon solutions of [10] to obtain those given in (41). In addition, apply the same change of variables to the metric in [10] to obtain the metric $\widetilde{G}_{i j}$ :

$$
\widetilde{G}_{i j}=\left(\begin{array}{ccc}
1 & 0 & 0 \\
0 & r^{2} & 0 \\
0 & 0 & r^{2} n_{m}^{2} \sin ^{2} \theta
\end{array}\right) .
$$

Here, $r \in[0, \infty], \theta \in[0, \pi]$, and $\phi \in[0,2 \pi]$. This generalizes the results of [10] which only applies to dyons with one unit of magnetic charge; that is, $g_{m}=1 / g_{D 3}$.

The solutions $W(r), Q(r)$, and $S(r)$ are obtained as in [10]

$$
\begin{aligned}
& W(r)=w(x)=1-\frac{x}{\sinh x}, \\
& Q(r)=q(x)=\operatorname{coth} x-\frac{1}{x}, \\
& S(r)=s(x)=q(x)=\operatorname{coth} x-\frac{1}{x},
\end{aligned}
$$

where the dimensionless variable $x$ is related to the radial coordinate $r$ :

$$
x=\sin \psi g_{D 3} v \alpha_{1} r
$$

The field tensor $F_{A B}$ of a dyon with electric charge $g_{e}$ and magnetic charge $g_{m}$,

$$
g_{m}=\frac{n_{m}}{g_{D 3}},
$$

can now be obtained from (41). Specifically,

$$
\begin{aligned}
F_{t r} & =\frac{g_{e}}{g} S^{\prime}(r) g_{D 3} v \alpha_{1} T_{r}, \\
F_{t \theta} & =[1-W(r)] \frac{g_{e}}{g} S(r) g_{D 3} v \alpha_{1} T_{\theta}, \\
F_{t \phi} & =[1-W(r)] \frac{g_{e}}{g} S(r) g_{D 3} v \alpha_{1} n_{m} \sin \theta T_{\phi}, \\
F_{r \theta} & =-W^{\prime}(r) T_{\phi}, \\
F_{\phi r} & =-W^{\prime}(r) n_{m} \sin \theta T_{\theta}, \\
F_{\theta \phi} & =-W(r)(2-W(r)) n_{m} \sin \theta T_{r}, \\
D_{r} A_{4} & =Q^{\prime}(r) g_{D 3} v \alpha_{1} T_{r}, \\
D_{\theta} A_{4} & =[1-W(r)] Q(r) g_{D 3} v \alpha_{1} T_{\theta}, \\
D_{\phi} A_{4} & =[1-W(r)] Q(r) g_{D 3} v \alpha_{1} n_{m} \sin \theta T_{\phi} .
\end{aligned}
$$

We now show that gauge invariance of action, (23), implies $S L(2, Z)$ invariance. Consider $U(1)$ gauge transformations which are constant at infinity and are also rotations about the axis $\widehat{A}_{4}=A_{4} /\left|A_{4}\right|$, specifically the gauge transformations [8]

$$
\delta A_{\mu}^{a}=\frac{1}{g_{D 3} v \alpha_{1}}\left(\mathscr{D}_{\mu} A_{4}\right)^{a} .
$$

Action (23) is invariant under these gauge transformations. According to the Noether method, the generator of these gauge transformations, $\mathcal{N}$, is given by

$$
\mathcal{N}=\frac{\partial \mathscr{L}}{\partial \partial_{0} A_{\mu}^{a}} \delta A_{\mu}^{a} .
$$

Substituting the Lagrangian density (24) into (52), we obtain

$$
\mathcal{N}=\frac{\mathscr{G}_{e}}{g_{D 3}}+\frac{g_{D 3}^{2} \theta \mathscr{G}_{m}}{8 \pi^{2}},
$$

where

$$
\begin{aligned}
& \mathscr{G}_{m}=\frac{1}{v \alpha_{1}} \int d^{3} \xi \operatorname{STr}\left\{\mathscr{D} A_{4} \cdot B\right\}, \\
& \mathscr{G}_{e}=\frac{1}{g_{D 3}^{2} v \alpha_{1}} \int d^{3} \xi \operatorname{Tr}\left\{\mathscr{D} A_{4} \cdot P_{0}\right\}
\end{aligned}
$$

are the magnetic and electric charge operators. Since rotations of $2 \pi$ about the axis $\widehat{A}_{4}$ must yield the identity for physical states, that is,

$$
e^{2 \pi i \mathcal{N}}=1,
$$


applying the $U(1)$ transformation on the left side of (55) to states in the adjoint representation of $S U(n)$, we find that the eigenstates of $\mathcal{N}$ are quantized with eigenvalue

$$
\mathcal{N}=\alpha_{1} \eta
$$

where $\eta$ is an arbitrary integer. Substituting (56) into (53), we obtain

$$
g_{e}=\alpha_{1}\left[\eta g_{D 3}-\frac{\theta^{\prime}}{2 \pi} n_{m} g_{D 3}\right],
$$

where we have defined $\theta^{\prime}$ by

$$
\theta \equiv \alpha_{1} \theta^{\prime}
$$

and used the fact that

$$
g_{m} g_{D 3}=n_{m} 4 \pi
$$

Taking $\theta^{\prime}=0$ in (57), we obtain the quantization condition for the electric charge

$$
g_{e}=\eta \alpha_{1} g_{D 3}
$$

The electromagnetic contribution to the mass (rest energy) of the dyon, $m_{\mathrm{em}}$, can be obtained by substituting (54a) and (54b) into (39) and integrating the second term within the trace to obtain

$$
m_{\mathrm{em}}=v \alpha_{1} \sqrt{g_{e}^{2}+g_{m}^{2}}
$$

We can now make $S L(2, Z)$ symmetry explicit. We first define

$$
\tau=\frac{\theta^{\prime}}{2 \pi}+\frac{4 \pi}{g_{D 3}^{2}} i .
$$

If $\theta^{\prime}=0$, then the weak/strong duality condition $g_{D 3} \rightarrow$ $g_{m}=(4 \pi) / g_{D 3}$ is equivalent to

$$
\tau \longrightarrow-\frac{1}{\tau}
$$

In (57), the transformation $\theta^{\prime} \rightarrow \theta^{\prime}+2 \pi$ results in identical physical systems with only states being relabeled. The transformation is equivalent to

$$
\tau \longrightarrow \tau+1
$$

Transformations (63) and (64) generate the group $S L(2, Z)$. See $[8,9]$ for further details.

Note that in (54a) and (54b) $\mathscr{G}_{e}$ is, strictly speaking, not the electric charge operator because $P_{0}$ is not the electric field but rather is its conjugate; however, according to (33) and (34), if $\mathscr{D} A_{4}$ and $B$ become vanishingly small for asymptotically large values of the radial coordinate, then $P_{0}$ approaches $E$. Thus, in the asymptotic limit $\mathscr{G}_{e}$ is the electric charge operator. This distinguishing feature is a direct consequence of the fact that our analysis is based on the BornInfeld action rather than the Yang-Mills-Higgs action. In our case, this point is inconsequential since $P_{0}=E$, exactly.
2.2. Dyon Solutions on D3-Branes. As emphasized previously, the dyon solutions derived in Section 2.1, when interpreted from $3+1$ spacetime dimensions, that is, the compactified theory in which $R_{4} \rightarrow 0$, are the 't Hooft/Polyakov magnetic monopole or dyon, with the potential $A_{4}$ being a Higgs boson transforming in the adjoint representation of the gauge group $U(n)$. Here, our purpose is to reinterpret these dyon solutions in which $R_{4} \rightarrow 0$ from the equivalent $T$-dual theory. In the $T$-dual theory, the radius $R_{4}$ is replaced by $R_{4}^{\prime}\left(R_{4}^{\prime}=\alpha^{\prime} / R_{4}\right)$ so that the radius of the $x^{4}$-dimension $R_{4}^{\prime} \rightarrow \infty$. In addition, the potential $A_{4}$ is reinterpreted as the $x^{4}$-coordinates of the $n$ D3-branes embedded in $4+1$ dimensional spacetime. These coordinates can be directly obtained by diagonalizing $A_{4}$, (41), using a local gauge transformation which rotates $T_{r}$ into $T_{z}$. The $n x^{4}$-coordinates are the diagonal elements of the matrix; that is (we are assuming that after the $T$-duality transformation the D3-branes are far from any orientifold hyperplanes. This can always be accomplished by adding the to $A_{4}$ component of the gauge potential a constant $U(1)$ gauge transformation $\theta_{0} T^{0}, \theta_{0}$ being a suitable constant (see Appendix A)),

$$
\begin{aligned}
& A_{4} \longrightarrow 2 \pi \alpha^{\prime} g_{D 3} v\left[\alpha_{2} T_{\perp}+Q(r) \alpha_{1} T_{z}\right] \\
& =2 \pi \alpha^{\prime} g_{D 3} v\left(\begin{array}{ccccc}
u_{1} & 0 & \cdots & 0 & 0 \\
0 & \ddots & \vdots & \vdots & \vdots \\
\vdots & \cdots & u_{1} & 0 & 0 \\
0 & \cdots & 0 & u_{2}+\tilde{u}(r) & 0 \\
0 & \cdots & 0 & 0 & u_{2}-\tilde{u}(r)
\end{array}\right),
\end{aligned}
$$

where

$$
\begin{aligned}
u_{1} & =-\frac{\alpha_{2}}{\sqrt{n(n-2)}}, \\
u_{2} & =\frac{\alpha_{2}}{2} \sqrt{\frac{n-2}{n}}, \\
\tilde{u}(r) & =\frac{\alpha_{1}}{2} Q(r) .
\end{aligned}
$$

Of the $n$ D3-branes $n-2$ of the D3-branes, denoted by $D 3_{n-2}$, are coincident. The $x^{4}$-coordinate of each is the constant value $2 \pi \alpha^{\prime} g_{D 3} v u_{1}$. For the remaining two D3branes, denoted by $D 3_{1}$ and $D 3_{2}$, the $x^{4}$-coordinate of each is a function of the radial coordinate $r$. Specifically, $x^{4}=$ $2 \pi \alpha^{\prime} g_{D 3} v\left(u_{2}-\tilde{u}(r)\right)$ for $D 3_{1}$ and $x^{4}=2 \pi \alpha^{\prime} g_{D 3} v\left(u_{2}+\widetilde{u}(r)\right)$ for $D 3_{2}$, and as a consequence these two D3-branes have nonvanishing intrinsic curvature. This occurs despite the fact that before the application of the $T$-duality transformation no gravitational interaction is explicitly present. We now introduce the length scale $L_{D 3}$ which is the separation between $D 3_{1}$ and $D 3_{2}$, in the asymptotic limit as the radial coordinate $r \rightarrow \infty$. It is related to previously defined parameters by

$$
L_{D 3}=2 \pi \alpha^{\prime} g_{D 3} v \alpha_{1} \text {. }
$$


Another relevant length scale is the size of the dyon, that is, the region of space where all components of the Yang-Mills field, $F_{A B}$, are nonvanishing. According to (49), (46a), (46b), (46c), and (47), only the radial components of the electric and magnetic fields are long range, with the remaining components of the fields vanishing exponentially for $x \gg 1$. Thus, additional structure of the dyon becomes apparent whenever $x \lesssim 1$ or equivalently whenever $r \lesssim 1 /\left(\sin \psi g_{D 3} v \alpha_{1}\right)$. We can therefore define the size of dyon $L_{d}$, as measured from asymptotically flat space, that is, $r \rightarrow \infty$, to be

$$
L_{d}=\frac{1}{\sin \psi g_{D 3} v \alpha_{1}}=\frac{2 \pi \alpha^{\prime}}{\sin \psi L_{D 3}} .
$$

In Figure 1, we show, for the gauge group $S U(5)$, embedding plots of the $5 \mathrm{D} 3$-branes as a function of the dimensionless radial coordinate, $x\left(x=r / L_{d}\right)$. As $x \rightarrow \infty$, the $x^{4}$ coordinate of $D 3_{2}$ approaches that of the (5-2) coincident $D 3$-branes, $D 3_{5-2}$, in effect, joining them by a wormhole in an asymptotically flat region of space. At $x=0, D 3_{2}$ is joined to $D 3_{1}$ by another wormhole. (See [11] for a recent discussion of thin shell wormholes exhibiting cylinder symmetry.) As we will show, in general, the intrinsic curvature of the two surfaces in the neighborhood of $x=0$ is relatively large but, nonetheless, finite. Although these features described in Figure 1 apply to the particular gauge group $S U(5)$, they apply to all $S U(n), n \geq 2$. (For $n=2$ there are no coincident D3branes.)

We now consider in detail the two D3-branes, $D_{1}$ and $D_{2}$, whose $x^{4}$-coordinates are radial dependent. The $T$-duality transformation on the $x^{4}$-dimension pulls back the metric onto $D_{1}$ and $D_{2}$, inducing the metric, $G_{i j}^{\prime}$,

$$
G_{i j}^{\prime}=\widetilde{G}_{i j} I_{n}+\left(\mathscr{D}_{i} A_{4}\right)^{(i)} T_{(i)} \mathscr{D}_{j} A_{4}^{(j)} T_{(j)} \delta_{(i)(j)} \widetilde{G}_{44} I_{n}
$$

Expanding the right-hand side of (69), we obtain

$$
G_{i j}^{\prime}=\left(\begin{array}{ccccc}
\widetilde{G}_{i j} & 0 & \cdots & 0 & 0 \\
0 & \ddots & \vdots & \vdots & \vdots \\
\vdots & \cdots & \widetilde{G}_{i j} & 0 & 0 \\
0 & \cdots & 0 & \widetilde{G}_{i j}+\widetilde{A}_{i j} & 0 \\
0 & \cdots & 0 & 0 & \widetilde{G}_{i j}+\widetilde{A}_{i j}
\end{array}\right) \text {, }
$$

where

$$
\begin{aligned}
\widetilde{A}_{i j}= & \left(\frac{g_{D 3} L \alpha_{1}}{2}\right)^{2} \\
& \times\left(\begin{array}{ccc}
{\left[Q^{\prime}(r)\right]^{2}} & 0 & 0 \\
0 & \widetilde{W}^{2}(r) & 0 \\
0 & 0 & \widetilde{W}^{2}(r) n_{m}^{2} \sin ^{2} \theta
\end{array}\right) .
\end{aligned}
$$

Here,

$$
\widetilde{W}(r)=[1-W(r)] Q(r),
$$

and $\widetilde{G}_{i j}$ is given by (45). In obtaining (70), we have used (50) and the fact that the matrices $T_{r}, T_{\theta}$, and $T_{\phi},(43 \mathrm{a}),(43 \mathrm{~b})$, and (43c), satisfy the relationship

$$
T_{r}^{2}=T_{\theta}^{2}=T_{\phi}^{2}=\left(\frac{1}{2}\right)^{2}\left(\begin{array}{ccccc}
0 & \cdots & 0 & 0 & 0 \\
\vdots & \ddots & \vdots & \vdots & \vdots \\
0 & \cdots & 0 & 0 & 0 \\
0 & \cdots & 0 & 1 & 0 \\
0 & \cdots & 0 & 0 & 1
\end{array}\right) .
$$

Each of the diagonal entries in the matrix $G_{i j}^{\prime}$ corresponds to the metric on one of the $n$ D3-branes obtained from the $T$-duality transformation. In the case of the first $n-2$ entries, corresponding to the $D 3$-branes $D 3_{n-2}$, the metric is flat. In the case of the last two entries corresponding to the D3-branes, $D 3_{1}$ and $D 3_{2}$, their geometries are identical and intrinsically curved. The only feature which distinguishes these two D3-branes is that the function $Q(r)$ defining the $x^{4}$ coordinate for $D 3$-brane $D_{2}$ is replaced by $-Q(r)$ for $D 3_{1}$, as evidenced in (65) and (66a) and (66b) and (66c) and also in Figure 1. As a consequence, the electric and magnetic charges of the dyon on $D_{2}$ are minus the values on $D_{1}$. The electric and magnetic field lines enter the wormhole from one $D 3$-brane and exit from the other. Figure 2 is an embedding diagram showing the $D 3$-branes $D_{1}$ and $D_{2}$ in the neighborhood of the radial coordinate $r=0$. As there is no event horizon surrounding $r=0$, the two D3-branes are joined by a wormhole at $r=0$.

Of particular interest is the intrinsic scalar curvature of $D 3_{1}$ and $D 3_{2}$, in the neighborhood of $r=0$. The scalar curvature can be calculated from the metric $G_{i j}^{\prime}$, (70), and its value $R(0)$ at $r=0$ is

$$
R(0)=216 \sin \psi \frac{L_{D 3}^{6} \sin ^{3} \psi}{\left[L_{D 3}^{4} \sin ^{2} \psi+\left(12 \pi \alpha^{\prime}\right)^{2}\right]^{2}} .
$$

For a given value of $\sin \psi, R(0)$ assumes its maximum value $\widetilde{R}(0)$ :

$$
\widetilde{R}(0)=\frac{27 \sqrt{3}}{8} \frac{\sin \psi}{\pi \alpha^{\prime}},
$$

when $L_{D 3}=\widetilde{L}_{D 3}$, where

$$
\widetilde{L}_{D 3}=\left(\frac{12 \sqrt{3} \pi \alpha^{\prime}}{\sin \psi}\right)^{1 / 2} .
$$

For either $L_{D 3} \rightarrow 0$ or $L_{D 3} \rightarrow \infty$, the scalar curvature $R(0) \rightarrow 0$; that is, the geometry of $D_{1}$ and $D_{2}$ becomes flat, everywhere. The expression for the scalar curvature $R(r)$ is a complicated function of $r$ and not amenable to straightforward interpretation and, therefore, will not be given. In Figure 3, we show a plot of the scalar curvature as a function of the radial coordinate. In this example, $L_{D 3}=\sqrt{\alpha^{\prime}}$, and the dyon has only one unit of magnetic charge so that $\sin \psi=1$. Near $r=0$, the scalar curvature is positive and 


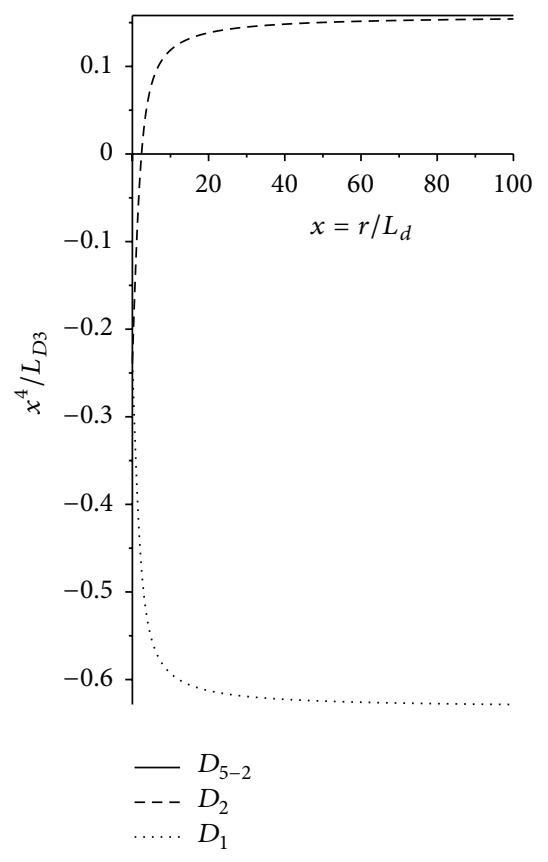

FIGURE 1: Embedding Functions of the 5 D3-branes for the gauge group $S U(5)$. The scaled coordinate $x^{4} / L_{D 3}$ is plotted as a function of the scaled radial coordinate, $r / L_{d}$, for the $5 D 3$-branes. The radial coordinate, $r$, is scaled by the size of the dyon, $L_{d}$, and the $x^{4}$ coordinate is scaled by the separation between the two D3-branes $D 3_{1}$ and $D 3_{1}$ in the asymptotic limit of large $r$.

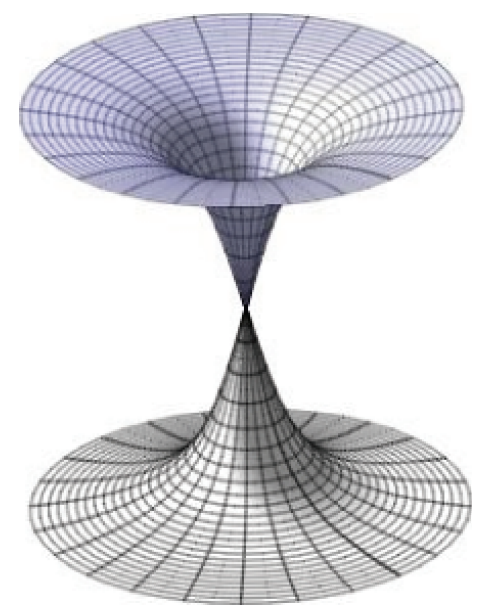

FIGURE 2: Wormhole. Shown is the embedding diagram for the two $D 3$-branes, $D 3_{1}$ and $D 3_{2}$, with azimuthal angle suppressed. The domain of the radial coordinate, $r$, is $0 \leq r \leq 15 L_{d}$, and the range of the embedding coordinate $x^{4}$ is $-.45 L_{D 3} \leq x^{4} \leq+.45 L_{D 3}$.

finite. As $r$ increases, the scalar curvature becomes slightly negative and asymptotically approaches zero as $r \rightarrow \infty$. These features of the scalar curvature described for this specific example also apply in general.

Consider dyon solutions for which $L_{D 3} \approx \sqrt{\alpha^{\prime}}$ or less. The $F$-strings connecting $D 3_{1}$ and $D 3_{2}$ would be in their ground state, a BPS state. In addition, assume that $g_{D 3} \ll 1$; then as $r \rightarrow 0$ from an asymptotically flat region of space, within

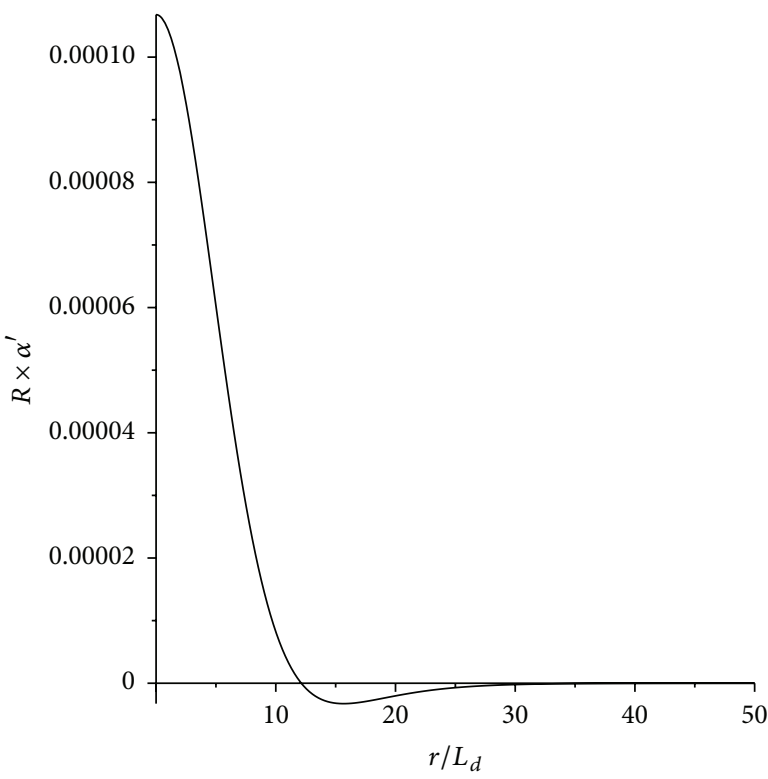

FIGURE 3: Scalar curvature $R(r)$. For the case $L_{D 3}=\sqrt{\alpha^{\prime}}$ and $\sin \psi=$ 1 , the dimensionless scalar curvature, $R \times \alpha^{\prime}$, is plotted as a function of the scaled radial coordinate, $r / L_{d}$.

either $D 3_{1}$ or $D 3_{2}$, the string length scale will be reached before the gravitational interaction becomes dominant at the length scale of $\mathcal{O}\left(g_{D 3}^{1 / 4} \sqrt{\alpha^{\prime}}\right)$ [5]. Thus, action, (2), which does not include the gravitational interaction, should apply, and consequently the dyon solutions derived should be accurate. On the other hand, let $g_{D 3} \rightarrow 1 / g_{D 3}$ so $L_{D 3} \gg 1$; then the $D$-string, also a BPS state, becomes lighter than the $F$-string. As a consequence of weak/strong duality, the dyon solutions should still be applicable with the $F$-strings being replaced by $D$-strings and the dyon electric and magnetic charges being interchanged.

After applying a $T$-duality transformation to the dyon solutions obtained in Section 2.1, we have obtained dyon solutions residing on $D 3$-branes where the effect of the gravitational interaction is apparent. This occurs despite the fact that action, (2), does not explicitly include the gravitational interaction. The presence of gravitational effects in this case is an example of how, in string theory, one-loop open string interactions, that is, Yang-Mills interactions, are related to tree level, closed string interactions, that is, gravitational interactions. In Figure 4, we depict, for illustrative purposes, two parallel $D p$-branes in close proximity. The two $D p$ branes can interact through open strings which connect the two $D p$-branes. In the figure, we show the one-loop vacuum graph for such an interaction which can be interpreted as an open string moving in a loop. Alternatively, the interaction can be interpreted as a closed string being exchanged between two $D p$-branes. In a certain sense, spin-2 gravitons, that is, the closed strings in their massless state, comprise a bound state of spin-one Yang-Mills bosons, that is, open strings in their massless state.

Prior to the application of the $T$-duality transformation, the open strings can propagate anywhere in the D4-brane. 


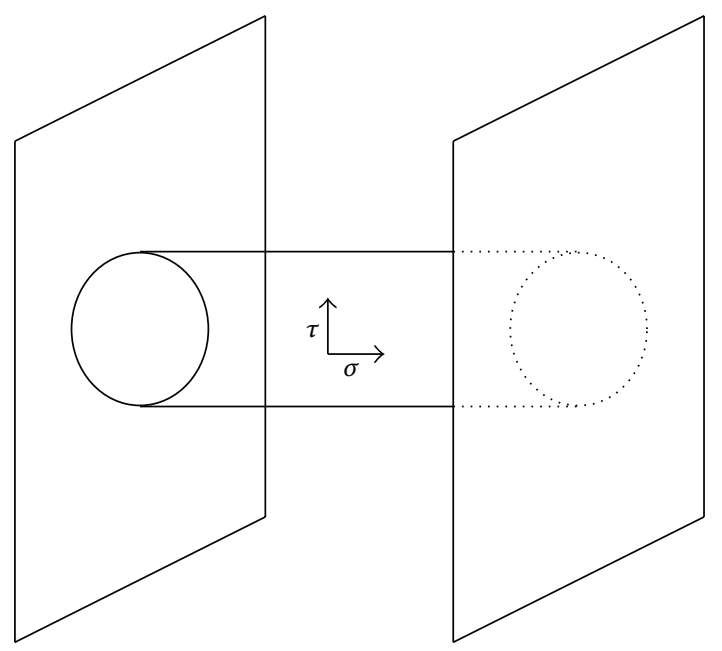

FIgURE 4: One-Loop String Diagram. Shown is the diagram for an open string, whose end points are fixed on two different $D p$ branes, propagating in a loop, $\tau$ being the time variable on the world sheet. Alternatively, interchanging $\tau$ with $\sigma$, the same diagram can be interpreted as a closed string being exchanged between the two Dp-branes.

After the $T$-duality transformation, the open strings are constrained to propagate only within D3-branes, whereas the closed strings can still propagate in the bulk region between the D3-branes; that is, gravitons can propagate in spacetime dimensions not allowed for the Yang-Mills bosons. Based on certain general assumptions, Weinberg and Witten have shown the impossibility of constructing a spin-2 graviton as a bound state of spin-1 gauge fields [12]. One of the assumptions on which the proof is based is that the spin1 gauge bosons and spin-2 gravitons propagate in the same spacetime dimensions. In the example presented here, this assumption is violated so that the conclusion of their theorem is avoided. These dyon solutions, thus, provide a simple example of gauge/gravity duality, which is discussed in detail in the work of Polchinski [13].

\section{Conclusions}

We have investigated dyon solutions within the context of superstring theory. Beginning with type I SO(32) superstring theory in ten dimensions, six of the spatial dimensions of which are compact, we have applied the group of $T$-duality transformations to five of the compact dimensions. The result is 16 D4-branes, a number $n(2 \leq n \leq 16)$ of which are coincident. The five $T$-dualized dimensions, whose size is taken to be vanishingly small, become the five internal spacetime dimensions while the remaining five dimensions correspond to the external $4+1$-dimensional spacetime. Making a suitable ansatz for the gauge fields residing on the $n$ coincident D4-branes, we have obtained dyon solutions from an action consisting of two terms: the $4+1$-dimensional, non-Abelian Dirac-Born-Infeld action and a Wess-Zuminolike action. The former action gives the low energy effective coupling of D4-branes to NS-NS closed strings and the latter of $D 4$-branes to $R-R$ closed strings. The method of solution involves transforming the $4+1$-dimensional action from the Lagrangian formalism to the Hamiltonian formalism and then seeking solutions which minimize the energy. The resulting dyon solutions, which are BPS states, reside on the $n$ D4-branes and are therefore associated with a supersymmetric $U(n)$ gauge theory in $4+1$ spacetime dimensions. These dyon solutions can be alternatively understood in the limit when the size of the remaining compact spacetime dimension, $x^{4}$, approaches zero. In this situation, the $4+1$ dimensional spacetime is reduced to a $3+1$-dimensional spacetime. As a consequence, the $A_{4}$ component of the vector potential becomes a Lorentz scalar with respect to $3+1$-dimensional spacetime and can be interpreted as a Higgs boson transforming as the adjoint representation of the $U(n)$ gauge group, analogous to the Higgs boson associated with the 't Hooft/Polyakov magnetic monopole. Finally, we perform a $T$-duality transformation in the $x^{4}$-direction. As a result, $n-2$ of the $D 4$-branes are transformed into $n-2$ coincident D3-branes, whose intrinsic geometry is flat. The remaining two D4-branes are transformed into two separate D3-branes whose intrinsic geometry is curved. As depicted in Figure 3, the two D3-branes are joined by wormhole at $r=0$. The scalar curvature of each D3-brane reaches a maximum, finite value, at $r=0$ and approaches zero as $r \rightarrow \infty$. The dyon resides on these two D3-branes. Furthermore, the values of electric and magnetic charges of the dyon on one D3-brane are minus the values on the other D3-brane, and as a consequence the electric and magnetic field lines enter the wormhole from one D3-brane and exit from the other D3brane.

The $T$-duality transformation in the $x^{4}$-direction causes two of the D3-branes to acquire intrinsic curvature. This occurs despite the fact that the Lagrangian density from which the dyon solutions have been obtained does not explicitly include the gravitational interaction. This can be understood heuristically from the open string, one-loop vacuum graph given in Figure 4 . From one perspective, the graph describes an open string, whose ends are fixed on two different D3-branes, moving in a loop, or, alternatively, the exchange of a closed string between two D3-branes. Thus, the gravitational interaction, that is, the closed string interaction, and the Yang-Mills interaction, that is, the open string interaction, appear as alternative descriptions of the same interaction. This simple example is suggestive of the subtle, but profound, connection between the Yang-Mills and gravitational interactions, specifically gauge/gravity duality.

\section{Appendices}

\section{A. Units and Conventions}

Concerning conventions, the Minkowski signature is $(-+$ $++\ldots)$, and the Levi-Cività symbols $\epsilon_{0123}=\epsilon_{123}=$ 1. Other relevant conventions are as follows: Greek letters denote four-dimensional spacetime indices, that is, $0,1,2$, and 3, whereas capitalized Roman letters are used when the spacetime dimension is greater than four. The small Roman letters, $i, j, k, l$, and $m$, are reserved for spatial 
dimensions in four-dimensional spacetime, that is, 1,2 , and 3. The small Roman letters, $a, b, c, d$, are used to enumerate the generators of the Lie group. The Levi-Cività tensor in three space $\operatorname{dimensions}$ is $\varepsilon_{i j k}=\sqrt{\left|\operatorname{det}\left(\widetilde{G}_{i j}\right)\right|} \epsilon_{i j k}$, where $\widetilde{G}_{i j}$ are the spatial components of the metric tensor. We focus our attention on Yang-Mills theories based on the compact Lie groups, $U(n)$. A typical group element $u(u \in U(n))$ is represented in terms of the group parameters $\theta_{a}(a=$ $\left.0 \cdots n^{2}-1\right)$ as $u=e^{i \theta_{a} T^{a}}$. The generators of this group in the fundamental representation are denoted by $T^{a}(a=$ $\left.0 \cdots n^{2}-1\right)$. The generator $T^{0}$ generates the $U(1)$ portion of $U(n)$, and the remaining $T^{a}$ generate the $S U(n)$ portion. The Lie algebra of the group generators, $T^{a}$, is $\left[T^{b}, T^{c}\right]=$ if ${ }^{a b c} T^{c}$, with $f^{a b c}$ being the structure constants of $U(n)$. The generators of $U(n)$ are required to satisfy the trace condition $\operatorname{Tr}\left(T^{a} T^{b}\right)=\delta^{a b} / 2$. Thus, in particular, $T^{0}=(1 / \sqrt{2 n}) I_{n}$, $I_{n}$ being the $n$-dimensional identity matrix. The Yang-Mills coupling constant is denoted by $g_{D 3}^{2}$. We employ LorentzHeaviside units of electromagnetism so that $c=\hbar=\epsilon_{0}=$ $\mu_{0}=1$; consequently, the Dirac quantization condition is $g_{D 3} g_{m}=2 \pi$. The quantity $g_{m}$ is the magnetic charge of a unit charged Dirac monopole.

Consistent with our analysis, the Yang-Mills-Higgs Lagrangian is

$$
\begin{aligned}
\mathscr{L}= & -\frac{1}{2 g_{D 3}^{2}} \operatorname{Tr}\left(F_{\mu \nu} F^{\mu \nu}\right)+\operatorname{Tr}\left(\mathscr{D}_{\mu} W \mathscr{D}^{\mu} W\right) \\
& -V(2 \operatorname{Tr}[W W]) \\
= & -\frac{1}{4 g_{D 3}^{2}} F_{\mu \nu}^{a} F^{a \mu \nu}+\frac{1}{2} \mathscr{D}_{\mu} W^{a} \mathscr{D}^{\mu} W^{a} \\
& -V\left(W^{a} W^{a}\right),
\end{aligned}
$$

where $V$ is a potential function depending on the Higgs field, $W$, and

$$
\begin{gathered}
F_{\mu \nu}=F_{\mu \nu}^{a} T^{a}, \\
W=W^{a} T^{a}, \\
A_{\mu}=A_{\mu}^{a} T^{a} .
\end{gathered}
$$

The covariant derivative, $\mathscr{D}_{\mu}$, is defined as

$$
\begin{aligned}
& \mathscr{D}_{\mu} \equiv \partial_{\mu}-i A_{\mu}, \\
& F_{\mu \nu}=-i\left[\mathscr{D}_{\mu}, \mathscr{D}_{\nu}\right] .
\end{aligned}
$$

Thus,

$$
F_{\mu \nu}^{a}=\partial_{\mu} A_{\nu}^{a}-\partial_{\nu} A_{\mu}^{a}+f^{a b c} A_{\mu}^{b} A_{\nu}^{c} .
$$

The Higgs field $W$ is a scalar transforming as the adjoint representation of $U(n)$ so that its covariant derivative is

$$
\mathscr{D}_{\mu} W=\partial_{\mu} W-i\left[A_{\mu}, W\right]=\partial_{\mu} W^{a}+f^{a b c} A_{\mu}^{b} W^{c} .
$$

\section{B. Relationships among the Various String Theory Parameters}

In principle, string theory has no adjustable parameters other than its characteristic length scale, $\sqrt{\alpha^{\prime}}$; however, various parameters of the theory do depend on values of the background fields. For reference, we provide explicit relationships between various string theory parameters and $\alpha^{\prime}$. Let $\Phi_{0}$ be the vacuum expectation value of the dilaton background; that is, $\phi_{0}=\langle\Phi\rangle$, $\Phi$ being the dilaton background. The closed string coupling constant, $g$, is

$$
g=e^{\Phi_{0}}
$$

The physical gravitational coupling, $\kappa$, is

$$
\kappa^{2} \equiv \kappa_{10}^{2} g^{2}=8 \pi G_{N}=\frac{1}{2}(2 \pi)^{7} \alpha^{\prime 4} g^{2},
$$

where $G_{N}$ is Newton's gravitational constant in 10 dimensions and $\kappa_{10}^{2}=\kappa_{11}^{2} / 2 \pi R$. The quantity $\kappa_{11}$ is the gravitational constant appearing in the eleven-dimensional, low energy effective action of supergravity, and $R$ is the compactification radius for reducing the eleven-dimensional theory to ten dimensions. The physical $D p$-brane tension, $\tau_{p}$, is

$$
\begin{aligned}
\tau_{p} & \equiv \frac{T_{p}}{g}=\frac{1}{g(2 \pi)^{p} \alpha^{\prime(p+1) / 2}} \\
& =\left(2 \kappa^{2}\right)^{-1 / 2}(2 \pi)^{(7-2 p) / 2} \alpha^{\prime 4},
\end{aligned}
$$

where $T_{p}$ is $D p$-brane tension. The $D p$-brane $R$ - $R$ charge, $\mu_{p}$, is

$$
\mu_{p}=g \tau_{p}=\frac{1}{(2 \pi)^{p} \alpha^{\prime(p+1) / 2}} .
$$

The coupling constant $g_{D p}$ of the $U(n)$ Yang-Mills theory on a $D p$-brane is given by

$$
g_{D p}^{2}=\frac{1}{\left(2 \pi \alpha^{\prime}\right)^{2} \tau_{p}}=(2 \pi)^{p-2} g \alpha^{\prime(p-3) / 2} .
$$

The ratio of the $F$-string tension, $\tau_{F 1}$, to the $D$-string ( $D 1$ brane) tension, $\tau_{D 1}$, is

$$
\frac{\tau_{F 1}}{\tau_{D 1}}=g
$$

\section{Evaluation of the Dirac-Born-Infeld Determinant in an Arbitrary Number of Dimensions}

In this appendix, we provide a heuristic derivation of the formula for evaluating an $N^{\prime}$-dimensional $\left(N^{\prime}=p+\right.$ 1) determinant of the form $\operatorname{det}\left(\widetilde{G}_{A B}+2 \pi \alpha^{\prime} F_{A B}\right)(A, B=$ $0 \cdots p),(2)$ and (5). (The notation used in this appendix does not adhere strictly to the font conventions defined in Appendix A.) Without loss of generality, we assume that 
the metric is diagonal. Consequently, we can express the determinant in the generic form

$$
\operatorname{det}(h) \epsilon_{I J \cdots O P}=\epsilon_{i j \cdots o p} h_{i I} h_{j J} \cdots h_{o O} h_{p P}
$$

where $h_{i j}=f_{i j}=2 \pi \alpha^{\prime} F_{(i-1)(j-1)},\left(i, j=1 \cdots N^{\prime}\right)$ and $h_{(i) i}=$ $g_{(i) i}=\widetilde{G}_{(i-1)(i-1)}$. Note: indices enclosed within parentheses are not summed.

Thus, the right-hand side of (C.1) comprises a sum of terms, each of which consists of products of the metric, $g_{(i) i}$ or $f_{i j}$. We need only to consider terms in which the number of $f_{i j}$ in each product is even, since terms containing products of an odd number of $f_{i j}$ vanish because $F_{A B}=-F_{B A}$. Thus, $\operatorname{det}\left(h_{i j}\right),($ C.1 $)$, can be reexpressed as a sum

$$
\begin{aligned}
\operatorname{det} & (h) \epsilon_{I J \cdots O P} \\
= & \left(H_{0}+H_{2}+H_{4}+H_{2 n^{\prime}}+\cdots\right) \epsilon_{I J \cdots O P} .
\end{aligned}
$$

Each $H_{2 n^{\prime}}$ is a term in (C.1) which contains a product of $f_{i j}$ which is even in number. The values of $2 n^{\prime}$ range from 0 to $N^{\prime}$ or $N^{\prime}-1$ depending on whether $N^{\prime}$ is even or odd. The value of $H_{0}$, which contains no off-diagonal elements, $f_{i j}$, is evaluated as

$$
H_{0}=\operatorname{det}(g) \equiv g_{11} g_{22} \cdots g_{N^{\prime} N^{\prime}}
$$

In order to understand the structure of $H_{2 n^{\prime}}$, for arbitrary $n^{\prime}$, we first study the structure of $H_{4}$ :

$$
\begin{gathered}
H_{4} \epsilon_{I J \cdots \mathrm{OP}}=\epsilon_{I J \cdots k l m n \cdots \mathrm{OP}} g_{(I) I} g_{(J) J} \\
\cdots g_{(O) O} g_{(P) P} f_{k K} f_{l L} f_{m M} f_{n N}
\end{gathered}
$$

Equation (C.4) represents a term in (C.1) where the values of $(k, l, m, n)$ in the sum are restricted to the specific integer values $(K, L, M, N)$ from the set of integers $\left(1,2 \cdots N^{\prime}\right)$. Multiplying (C.4) by the Levi-Cività symbol (which does not change value of the expression), we obtain

$$
\begin{aligned}
H_{4} & =g_{(I) I} g_{(J) J} \\
& \cdots g_{(O) O} g_{(P) P} \epsilon_{I J \cdots k l m n \cdots O P} f_{k K} f_{l L} f_{m M} f_{n N} \epsilon_{I J \cdots K L M N \cdots O P} .
\end{aligned}
$$

We now rearrange the terms in (C.5) in a form which is more useful for the subsequent analysis. By virtue of the LeviCività symbols, none of the $(k, l, m, n)$ is equal to any of the others, and similarly for $(K, L, M, N)$; however, each of the $(k, l, m, n)$ is equal to one of the $(K, L, M, N)$. Because of the antisymmetry of $f_{i j}$, some of the terms in the sum vanish, that is, whenever $k=K$, or whenever $l=L$, and so forth. By explicit construction or by a combinatorics argument, we can show that there are nine terms which are nonvanishing. We consider one typical term in the sum, for example, the term, $\{1\}$,

$$
\{1\}=\{m=K, k=L, n=M, l=N\} .
$$

We now show how to reexpress (C.5) so half, that is, 2 , of the four $f_{i j}$ are associated with one of the Levi-Cività symbols and the other half are associated with the other Levi-Cività symbol. In order for the two $f_{i j}$ to be associated with one Levi-Cività symbol, the four subscripts on the two $f_{i j}$ must be different. We associate the first $f_{k K}$ with the Levi-Cività symbol to its left. To determine the remaining associations, we proceed as follows. Since $K=m$, we associate $f_{m M}$ with the Levi-Cività symbol to the right. We now consider the second term in (C.5), that is, $f_{l L}$. Since $L=k$, we associate $f_{l L}$ with the Levi-Cività symbol to its right and $f_{n N}$ with the LeviCività symbol to the right. We continue in this manner to the next remaining term, $f_{n N}$. Since $N$ does not equal any of the lowercase Roman subscripts associated with the Levi-Cività symbol to the left $(N=l)$, we assign $f_{n N}$ to the Levi-Cività symbol to the left. This completes the process since each $f_{i j}$ is associated with either of the two Levi-Cività symbols. Using (C.6) we reexpress the subscripts on the Levi-Cività symbols in (C.5):

$$
\begin{aligned}
H_{4,\{1\}} & =g_{(I) I} g_{(J) J} \\
& \cdots g_{(O) O} g_{(P) P} \epsilon_{I J \cdots(k) N K(n) \cdots O P} f_{k K} f_{n N} f_{m M} f_{l L} \epsilon_{I J \cdots(m) L M(l) \cdots O P} .
\end{aligned}
$$

Now, we permute the subscripts of the Levi-Cività symbols so that the corresponding lowercase and uppercase Roman letters are adjoining. Both uppercase $M$ and $K$ require the same number of movements as the uppercase $N$ and $L$ do. Since the number of permutations is even, no change in sign of the Levi-Cività symbols results from permuting the subscripts. Equation (C.7) becomes

$$
\begin{aligned}
& H_{4,\{1\}}=g_{(I) I} g_{(J) J} \\
& \quad \cdots g_{(O) O} g_{(P) P} \epsilon_{I J \cdots(k)(K)(n)(N) \cdots \mathrm{OP}} f_{k K} f_{n N} f_{m M} f_{l L} \epsilon_{I J \cdots(m)(M)(l)(L) \cdots \mathrm{OP} .}
\end{aligned}
$$

Each of the remaining $H_{4,\{s\}}(s=2 \cdots 9)$ can be expressed, similarly.

In order to understand, in generic terms, the structure of $\mathrm{H}_{4}$, consider the following expression $\mathrm{H}_{4}^{\prime}$ :

$$
\begin{aligned}
H_{4}^{\prime} & =g_{(I) I} g_{(J) J} \\
& \cdots g_{(O) O} g_{(P) P} \epsilon_{I J \cdots k K n N \cdots O P} f_{k K} f_{n N} f_{m M} f_{l L} \epsilon_{I J \cdots m M l L \cdots O P} .
\end{aligned}
$$

The expression $H_{4}^{\prime}$ differs from the individual term $H_{4,\{1\}}$ in that the repeated indices $(k, K, n, N)$ and $(m, M, l, L)$ are summed. By inspection, the term $H_{4,\{1\}}$, as well as the remaining terms $H_{4,\{s\}}(s=2 \cdots 9)$, is contained in $H_{4}^{\prime}$. Overtly, the number of nonvanishing terms in (C.9) is $(4 !)^{2}$, each factor of (4!) coming from each one of the Levi-Cività symbols. By a combinatorics argument, each factor of 4 ! is eightfold redundant so that the number of independent terms associated with each Levi-Cività symbol is three. Consequently, the number of independent terms in $H_{4}^{\prime}$ is 9 , that is, $3 \times 3$, with redundancy $8 \times 8$. Thus,

$$
H_{4}=\frac{1}{8 \times 8} H_{4}^{\prime}
$$

Using similar reasoning, we can show that

$$
H_{2 n^{\prime}}=\frac{H_{2 n^{\prime}}^{\prime}}{R_{2 n^{\prime}}^{\prime} \times R_{2 n^{\prime}}^{\prime}},
$$


where

$$
R_{2 n^{\prime}}^{\prime}=2^{n^{\prime}} n^{\prime} !
$$

In order to show (C.11), one needs to use the fact that the number of nonvanishing terms, $R_{2 n^{\prime}}$, in $H_{2 n^{\prime}}$ is given by

$$
\begin{aligned}
R_{2 n^{\prime}} & =\left(2 n^{\prime}-1\right) R_{2\left(n^{\prime}-1\right)}\left(2 n^{\prime}-1\right) \\
& =\left[\left(2 n^{\prime}-1\right) ! !\right]^{2},
\end{aligned}
$$

so that the number of independent terms, $r_{2 n^{\prime}}$, associated with each of the two Levi-Cività symbols of $H_{2 n^{\prime}}^{\prime}$ is

$$
r_{2 n^{\prime}}=\left(2 n^{\prime}-1\right) ! !
$$

Thus,

$$
R_{2 n^{\prime}}^{\prime}=\frac{\left(2 n^{\prime}\right) !}{r_{2 n^{\prime}}}=2^{n^{\prime}} n^{\prime} !
$$

Both (C.13) and (C.14) are obtained from combinatorics arguments. Using the metric tensor to lower indices in $f^{i j}$ and the relationship (C.15), we reexpress (C.11)

$$
\begin{aligned}
H_{2 n^{\prime}}= & -\operatorname{det}(g) \frac{1}{\left(N^{\prime}-2 n^{\prime}\right) !}\left(2 n^{\prime}-1\right) ! !^{2} \\
& \times *(\underbrace{f \wedge f \wedge \cdots f}_{n^{\prime}}) \\
& *(\underbrace{f \wedge f \wedge \cdots \wedge f}_{n^{\prime}}) .
\end{aligned}
$$

The Hodge $*$ operation transforms an $s$-form $Q$ in an $N^{\prime}$ dimensional space to an $\left(N^{\prime}-s\right)$-form whose components are

$$
\begin{aligned}
\left({ }^{*} Q\right)_{i_{s+1} \cdots i_{N^{\prime}}} & =Q^{i_{j_{1}} \cdots j_{s}} \frac{1}{s !} \sqrt{\operatorname{det}|g|} \epsilon_{j_{1} j_{2} \cdots j_{s} i_{s+1} \cdots i_{N^{\prime}}}, \\
{ }^{*} Q \cdot{ }^{*} Q & \equiv\left({ }^{*} Q\right)_{i_{s+1} \cdots i_{N^{\prime}}}\left({ }^{*} Q\right)^{i_{s+1} \cdots i_{N^{\prime}}} .
\end{aligned}
$$

Note that in (C.16) $\underbrace{f \wedge f \wedge \cdots f}_{n^{\prime}}$ is a $2 n^{\prime}$-form. Also, in (C.16), the minus sign to the right of the equal sign is a consequence of the Minkowski signature of the metric; that is, $\epsilon^{12 \cdots N^{\prime}}=$ $-\epsilon_{12 \cdots N^{\prime}}$. For Euclidean signature, the minus sign is replaced by a plus sign. Using properties of the Levi-Cività symbol, we can show that

$$
H_{2}=\operatorname{det}(g) \frac{1}{2 !} f_{i j} f^{i j}
$$

irrespective of the signature of the metric. Using (C.2), (C.16), and (C.18), we obtain

$$
\begin{aligned}
& \operatorname{det}(h)=\operatorname{det}(g)\left[1+\frac{1}{2 !} f_{i j} f^{i j}\right. \\
& \mp \sum_{2 n^{\prime}=4}^{2 n^{\prime}=N^{\prime \prime}}\left(\frac{1}{\left(N^{\prime}-2 n^{\prime}\right) !}\right. \\
& \times\left(2 n^{\prime}-1\right) ! !^{2 *}(\underbrace{f \wedge f \wedge \cdots f}_{n^{\prime}}) \\
& \left.\left.*(\underbrace{f \wedge f \wedge \cdots f}_{n^{\prime}})\right)\right]
\end{aligned}
$$

where

$$
N^{\prime \prime}= \begin{cases}N^{\prime} & \text { if } N^{\prime} \text { is even } \\ N^{\prime}-1 & \text { if } N^{\prime} \text { is odd. }\end{cases}
$$

The minus (plus) sign corresponds to a metric with Minkowski (Euclidean) signature.

For the case when $N^{\prime}=5$ and the metric has Minkowski signature, (C.19) reduces to

$$
\begin{aligned}
& \operatorname{det}(h)=\operatorname{det}(g)\left[1+\frac{1}{2 !} f_{i j} f^{i j}-\frac{1}{(5-2 \cdot 2) !}\right. \\
& \left.\times\left(3^{2} \cdot 1^{2}\right)^{*}(f \wedge f)_{k}{ }^{*}(f \wedge f)^{k}\right] .
\end{aligned}
$$

\section{Conflict of Interests}

The author declares that there is no conflict of interests regarding the publication of this paper.

\section{References}

[1] P. A. M. Dirac, "Quantised singularities in the electromagnetic field," Proceedings of the Royal Society A: Mathematical, Physical and Engineering Sciences, vol. 133, no. 821, pp. 60-72, 1931.

[2] G. 't Hooft, "Magnetic monopoles in unified gauge theories," Nuclear Physics B, vol. 79, pp. 276-284, 1974.

[3] C. Montonen and D. Olive, "Magnetic monopoles as gauge particles?" Physics Letters B, vol. 72, no. 1, pp. 117-120, 1977.

[4] A. H. Guth, "Inflationary universe: a possible solution to the horizon and flatness problems," Physical Review D, vol. 23, no. 2, pp. 347-356, 1981.

[5] J. Polchinski, String Theory Volume II, Cambridge University Press, New York, NY, USA, 1998.

[6] C. V. Johnson, D-Branes, Cambridge University Press, New York, NY, USA, 2003.

[7] A. A. Tseytlin, "On non-abelian generalisation of the BornInfeld action in string theory," Nuclear Physics B, vol. 501, no. 1, pp. 41-52, 1997. 
[8] J. A. Harvey, "Magnetic monopoles, duality, and supersymmetry," http://arxiv.org/abs/hep-th/9603086v2.

[9] E. Witten, "Dyons of charge e $\theta / 2 \pi$," Physics Letters $B$, vol. 86, no. 3-4, pp. 283-287, 1979.

[10] E. A. Olszewski, "Dyons and magnetic monopoles revisited," Particle Physics Insights, vol. 5, pp. 1-12, 2012.

[11] M. R. Setare and A. Sepehri, "Stability of cylindrical thin shell wormhole during evolution of universe from inflation to late time acceleration," JHEP, vol. 2015, no. 3, article 079, 16 pages, 2015.

[12] S. Weinberg and E. Witten, "Limits of massless particles," Physics Letters. B, vol. 96, no. 1-2, pp. 59-62, 1980.

[13] J. Polchinski, "Introduction to gauge/gravity duality," http:// arxiv.org/abs/1010.6134v1. 

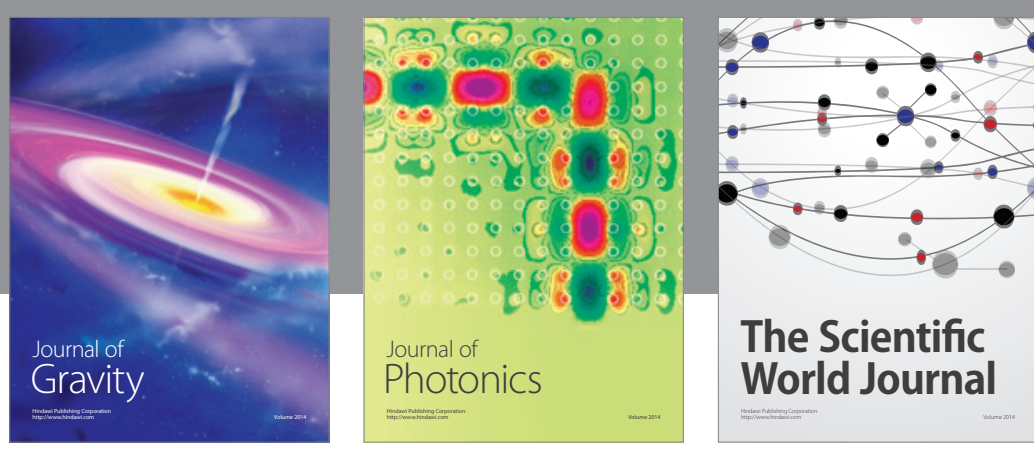

The Scientific World Journal
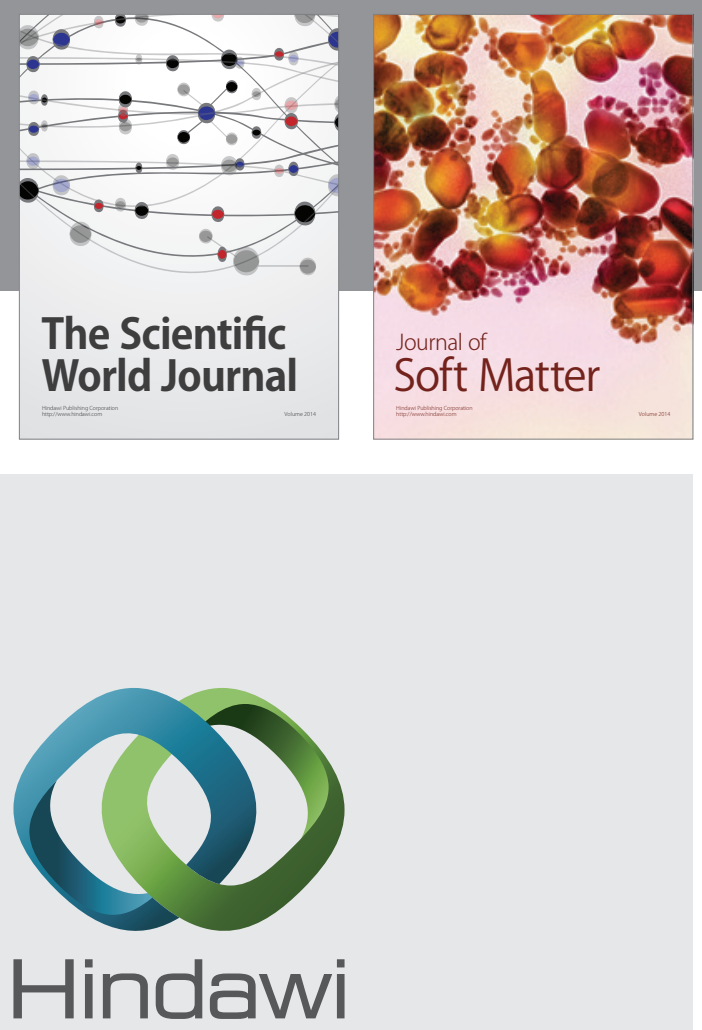

Submit your manuscripts at

http://www.hindawi.com

nternational Journal of

Statistical Mechanics
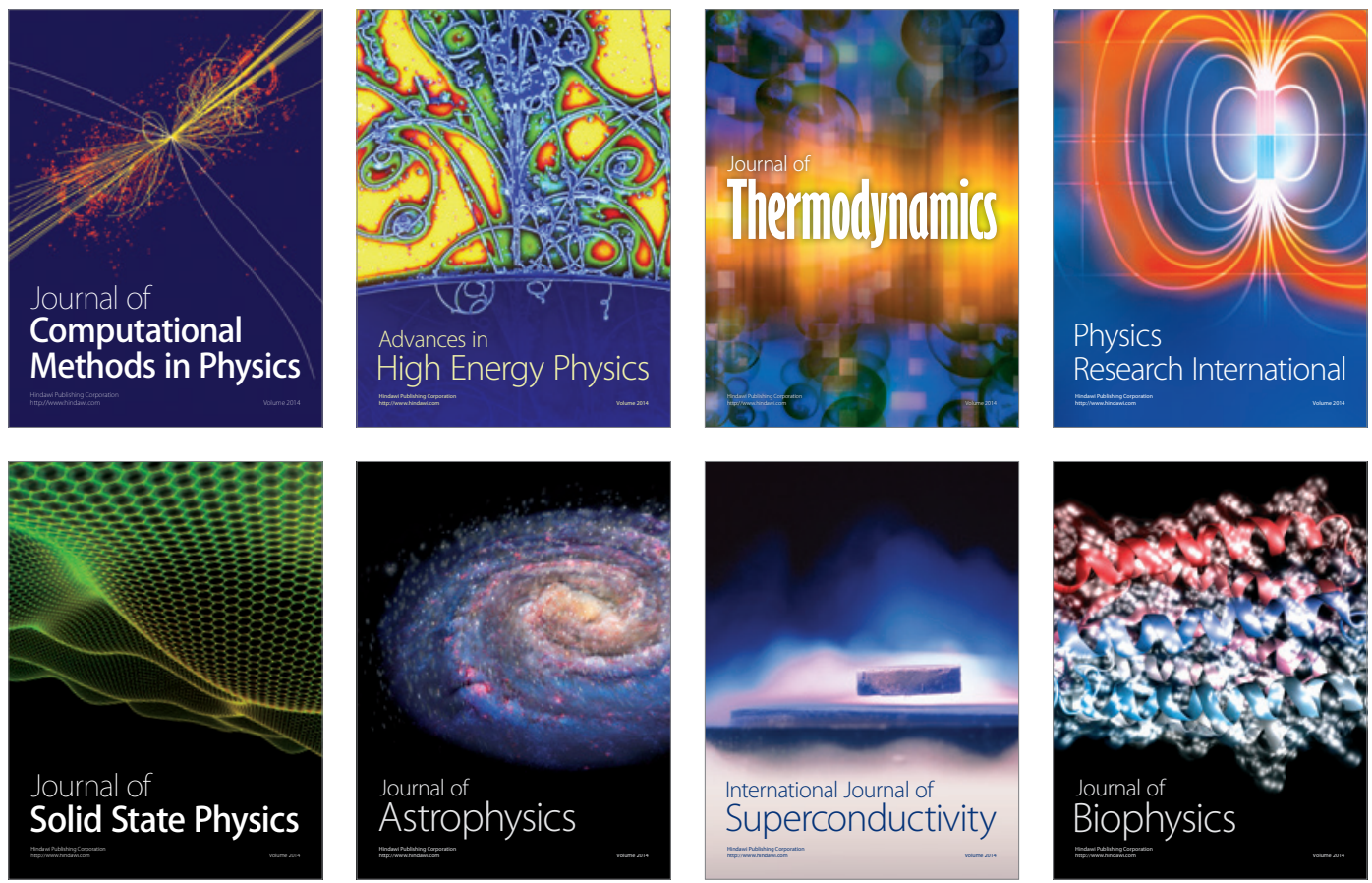
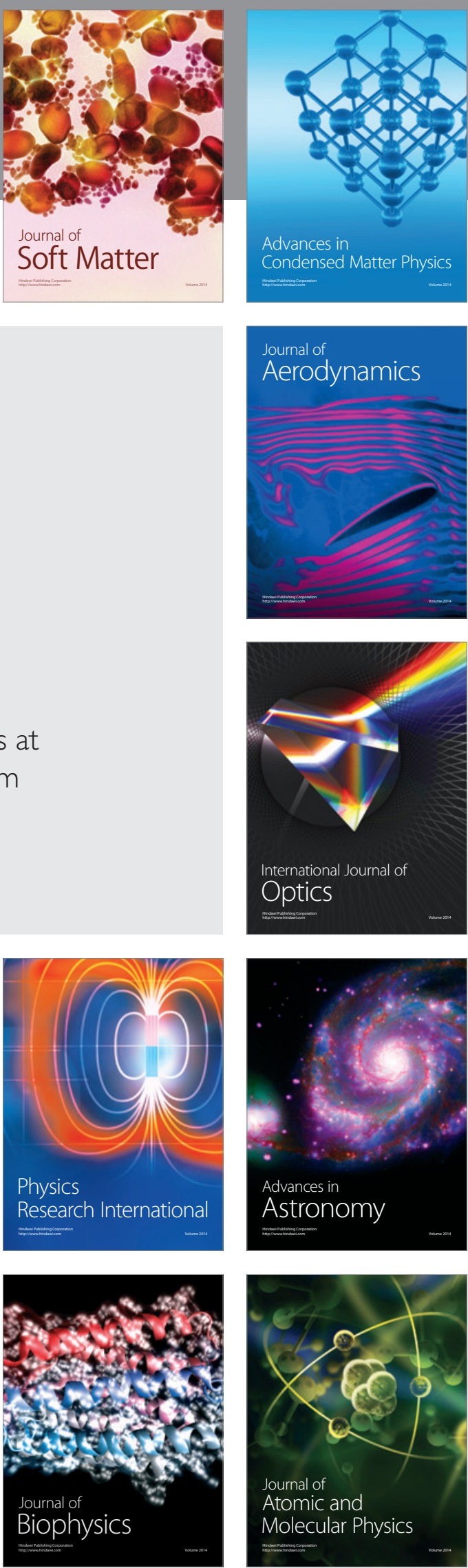